\title{
The HSP90 inhibitor ganetespib potentiates the antitumor activity of EGFR tyrosine kinase inhibition in mutant and wild-type non-small cell lung cancer
}

\author{
Donald L. Smith • Jaime Acquaviva • Manuel Sequeira • \\ John-Paul Jimenez • Chaohua Zhang • Jim Sang • \\ Richard C. Bates • David A. Proia
}

Received: 12 May 2014 / Accepted: 10 July 2014 / Published online: 1 August 2014

(C) The Author(s) 2014. This article is published with open access at Springerlink.com

\begin{abstract}
Small molecule inhibitors of epidermal growth factor receptor (EGFR) tyrosine kinase activity, such as erlotinib and gefitinib, revolutionized therapy for non-small cell lung cancer (NSCLC) patients whose tumors harbor activating EGFR mutations. However, mechanisms to overcome the invariable development of acquired resistance to such agents, as well as realizing their full clinical potential within the context of wild-type EGFR (WT-EGFR) disease, remain to be established. Here, the antitumor efficacy of targeted EGFR tyrosine kinase inhibitors (TKIs) and the HSP90 inhibitor ganetespib, alone and in combination, were evaluated in NSCLC. Ganetespib potentiated the efficacy of erlotinib in TKI-sensitive, mutant EGFR-driven NCI-HCC827 xenograft tumors, with combination treatment causing significant tumor regressions. In erlotinib-resistant NCI-H1975 xenografts, concurrent administration of ganetespib overcame erlotinib resistance to significantly improve tumor growth inhibition. Ganetespib co-treatment also significantly enhanced antitumor responses to afatinib in the same model. In WT-EGFR cell lines, ganetespib potently reduced cell viability. In NCIH1666 cells, ganetespib-induced loss of client protein expression, perturbation of oncogenic signaling pathways, and induction of apoptosis translated to robust single-agent activity in vivo. Dual ganetespib/erlotinib therapy induced regressions
\end{abstract}

Donald L. Smith and Jaime Acquaviva contributed equally to this manuscript.

Electronic supplementary material The online version of this article (doi:10.1007/s11523-014-0329-6) contains supplementary material, which is available to authorized users.

D. L. Smith · J. Acquaviva • M. Sequeira $\cdot$ J.-P. Jimenez $\cdot$

C. Zhang $\cdot$ J. Sang $\cdot$ R. C. Bates $\cdot$ D. A. Proia $(\bowtie)$

Synta Pharmaceuticals Corp, 125 Hartwell Ave, Lexington,

MA 02421, USA

e-mail: dproia@syntapharma.com in NCI-H322 xenograft tumors, indicating that the sensitizing properties of ganetespib for erlotinib were conserved within the WT-EGFR setting. Mechanistically, combined ganetespib/ erlotinib exposure stabilized EGFR protein levels in an inactive state and completely abrogated extracellular-signalregulated kinase (ERK) and AKT signaling activity. Thus, selective HSP90 blockade by ganetespib represents a potentially important complementary strategy to targeted TKI inhibition alone for inducing substantial antitumor responses and overcoming resistance, in both the mutant and WT-EGFR settings.

Keywords HSP90 inhibitor - Epidermal growth factor receptor · Non-small cell lung cancer · Ganetespib .

Combination therapy

\section{Introduction}

Lung cancer remains the leading cause of cancer mortality worldwide [1], with almost 160,000 deaths estimated for 2013 in the USA alone [2]. Non-small cell lung cancer (NSCLC) accounts for the majority ( $85 \%$ ) of all cases. Platinum-based combination chemotherapy provides the foundation for standard-of-care treatments in the clinical management of advanced NSCLC; unfortunately, this strategy has largely reached a plateau of effectiveness in improving overall survival rates for patients $[3,4]$. NSCLC is characterized by a remarkable degree of genetic diversity and may be further classified into distinct molecular subsets based on genotypic alterations that act as oncogenic drivers of malignancy in this disease [5, 6]. Over recent years, an increased biological understanding of NSCLC tumorigenesis has transformed lung cancer therapy from broad-based cytotoxic use toward more 
tailored treatment approaches for certain NSCLC patient populations [7]. This paradigm shift has, in turn, provided meaningful improvements in overall survival and quality of life [5, 7].

A prime example is provided by targeted inhibitors of the epidermal growth factor receptor (EGFR), which revolutionized and modified the principles of NSCLC treatment a decade ago [8]. EGFR is a member of the ErbB family of transmembrane receptor tyrosine kinases (EGFR/ErbB1, human epidermal growth factor receptor 2 (HER2)/ErbB2, HER3/ErbB3, and HER4/ErbB4). EGFR activation stimulates a variety of intracellular signaling cascades, including the JAK/signal transducers and activators of transcription (STAT), RAS/RAF/extracellular-signal-regulated kinase (ERK), and PI3K/AKT pathways [9]. In tumor cells, EGFR activity may become dysregulated through a number of mechanisms, including activating mutations, amplification of gene copy number, and/or receptor overexpression [10]. EGFR overexpression is a feature of NSCLC tumors, particularly in patients with metastatic disease, and is correlated with poor prognosis [11]. Gefitinib and erlotinib, both small-molecule EGFR tyrosine kinase inhibitors (TKIs), were the first molecularly targeted agents used in the treatment of advanced NSCLC. Dramatic tumor responses and favorable clinical outcomes led to their widespread use in the first-line setting for NSCLC patients bearing activating EGFR mutations. However, durable responses to either TKI are rare due to the invariable development of resistance, which commonly arises through the acquisition of a second-site mutation (T790M) within EGFR [12, 13], or via activation of compensatory signaling pathways that bypass the receptor and restore downstream oncogenic signaling [14]. Strategies to counteract acquired resistance to EGFR TKI therapy have not yet been established, and this remains an ongoing challenge for NSCLC treatment.

In addition, targeted EGFR therapy using erlotinib and gefitinib preceded a full understanding of their mechanism of action, such that regulatory approval that occurred before EGFR mutational status was identified as a predictive biomarker for clinical activity [15]. While the efficacy of these drugs is generally superior to chemotherapy for individuals whose tumors harbor EGFR mutations, the incidence of EGFR mutation-positive patients ranges from approximately $30 \%$ in Asian populations to only $10-15 \%$ in Western countries [16]. Thus, the clear majority of NSCLC patients worldwide do not harbor EGFR mutations, and the value and applicability of EGFR TKIs within the context of wild-type EGFR (WT-EGFR) NSCLC are controversial [17-19].

Selective inhibitors of the molecular chaperone heat shock protein 90 (HSP90) are an actively pursued class of agents currently under development as novel anticancer therapeutics [20]. HSP90 plays an essential role in regulating the functional stability and maturation of numerous key signal transduction proteins, termed "client" proteins [21]. Notably, a number of sensitive HSP90 clients have been implicated in the pathogenesis of NSCLC, including EGFR and anaplastic lymphoma kinase (ALK), and intermediates of oncogenic signaling cascades such as RAF and AKT [22]. Indeed, the chaperone activity of HSP90 is commonly exploited by cancer cells to facilitate multiple aspects of the tumorigenic process, including aberrant survival, oncogene addiction, and metastatic potential $[23,24]$. Because inhibition of HSP90 activity targets its clients for proteasomal destruction, pharmacological blockade of the chaperone provides a mechanism to concomitantly disrupt multiple oncogenic signaling cascades through a singular molecular target $[25,26]$. Moreover, this unique characteristic also serves as a means to overcome signaling redundancies and drug resistance mechanisms observed in many cancers [25-27].

Ganetespib is a potent, resorcinol-based small molecule inhibitor of HSP90 that exhibits robust preclinical activity against a range of cancer models, including NSCLC [28-31]. Importantly, beyond client-driven tumor types, ganetespib can potentiate the effects of other molecularly targeted and chemotherapeutic agents while simultaneously counteracting both intrinsic and acquired drug resistance in a variety of tumor models (reviewed in Proia and Bates [29]). Moreover, a maturing clinical profile has revealed evidence of therapeutic efficacy in NSCLC, most notably as a single agent in ALK-driven disease and as part of combination therapy with docetaxel in advanced adenocarcinoma patients [32, 33]. In light of these considerations, we sought to determine whether ganetespib could potentiate EGFR TKI activity within the context of EGFR mutant NSCLC, using both erlotinib-sensitive and erlotinib-resistant models. Since therapeutic response to HSP90 inhibition and EGFR kinase blockade is also of clinical interest for WT-EGFR lung cancer, we further combined these two modalities in NSCLC models of this phenotypic background. The data reported here suggest that ganetespib treatment alongside EGFR kinase inhibition offers a potentially important complementary strategy to targeted TKI inhibition alone for inducing substantial antitumor responses, as well as overcoming TKI resistance, in both the mutant and WTEGFR settings.

\section{Materials and methods}

Cell lines, antibodies, and reagents

The NCI-HCC827, NCI-H1975, NCI-H1395, NCI-H1666, and NCI-H292 cell lines were obtained from the American Type Culture Collection (ATCC, Manassas, VA, USA). NCI- 
H322 cells were purchased from Sigma-Aldrich (St. Louis, MO, USA). All were maintained at $37{ }^{\circ} \mathrm{C}$ in $5 \%(v / v) \mathrm{CO}_{2}$ using culture medium recommended by the supplier. The remaining NSCLC lines listed in Table 1 are part of a collection assembled by The Center for Molecular Therapeutics (Massachusetts General Hospital Cancer Center) who performed the drug sensitivity analysis. All primary antibodies were purchased from Cell Signaling Technology (CST, Beverly, MA, USA) with the exception of p-EGFR (Invitrogen, Grand Island, NY, USA) and the glyceraldehyde-3-phosphate dehydrogenase (GAPDH) antibodies (Santa Cruz Biotechnology Inc., Santa Cruz, CA). Ganetespib [3-(2,4-dihydroxy-5isopropylphenyl)-4-(1-methyl-1H-1,2,4-triazol-5(4H)-one] was synthesized by Synta Pharmaceuticals Corp. Erlotinib and afatinib were purchased from LC Laboratories (Woburn, MA, USA).

Table 1 In vitro cytotoxicity values of ganetespib in EGFR wild-type NSCLC lines

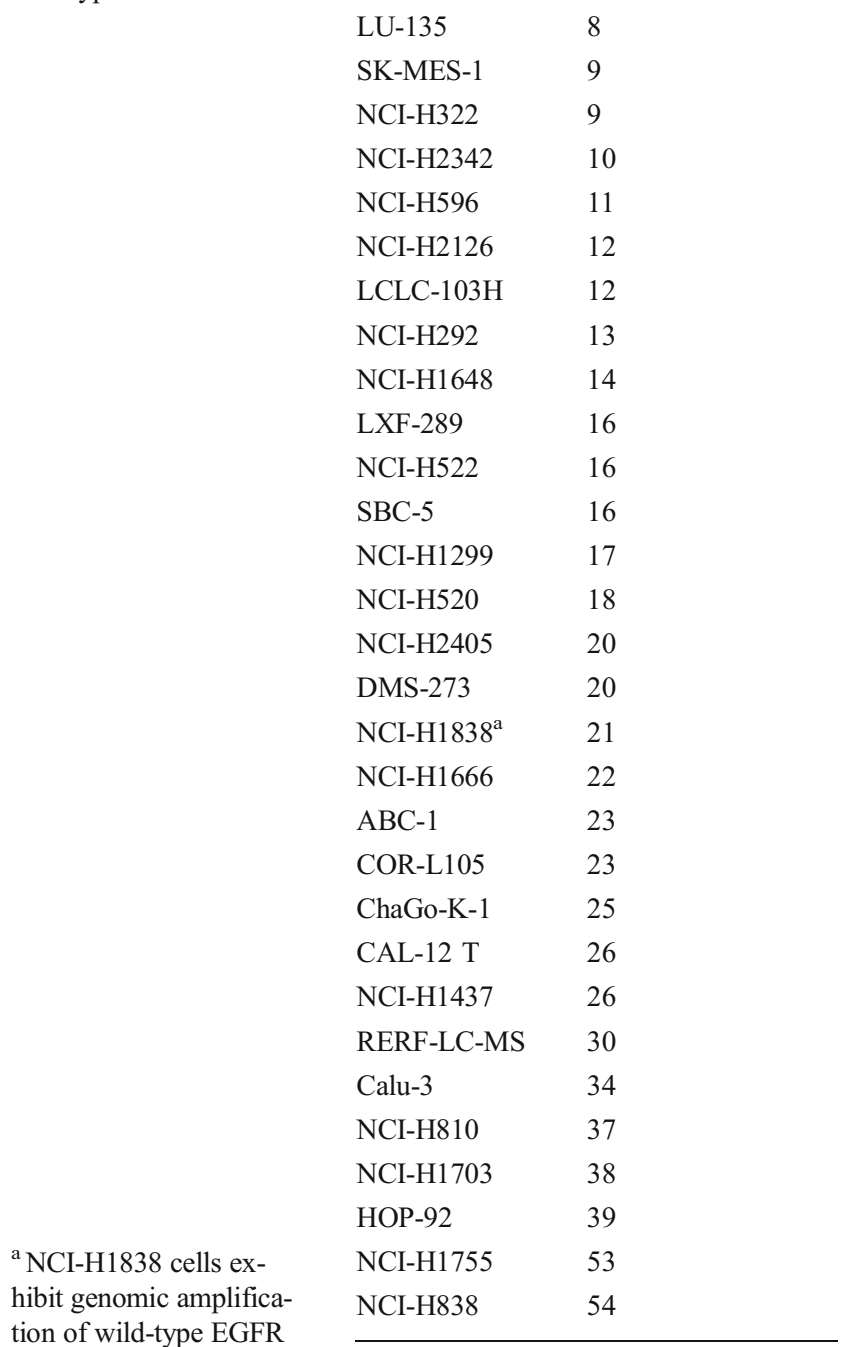

Cell viability assays

Cellular viability was assessed using the CellTiter-Glo Luminescent Cell Viability Assay (Promega, Madison, WI, USA) according to the manufacturer's protocol. Lung cancer cell lines were seeded into 96-well plates based on optimal growth rates determined empirically for each line. Twenty-four hours after plating, cells were dosed with graded concentrations of drug for $72 \mathrm{~h}$. CellTiter-Glo was added $(50 \% v / v)$ to the cells, and the plates incubated for $10 \mathrm{~min}$ prior to luminescent detection in a Victor 2 microplate reader (PerkinElmer, Waltham, MA, USA). Data were normalized to percent of control, and $\mathrm{IC}_{50}$ values were determined using XLfit software.

Western blotting

Following in vitro assays, tumor cells were disrupted in lysis buffer (CST) on ice for $10 \mathrm{~min}$. Lysates were clarified by centrifugation, and equal amounts of proteins resolved by SDS-PAGE before transfer to nitrocellulose membranes (Bio-Rad, Hercules, CA, USA). Membranes were blocked with StartingBlock T20 blocking buffer (Thermo Scientific, Cambridge, MA, USA) and immunoblotted with the indicated antibodies. Antibody-antigen complexes were visualized using an Odyssey system (LI-COR, Lincoln, NE, USA).

NSCLC xenograft tumor models

Female CB-17/severe combined immunodeficient (SCID) mice (Charles River Laboratories, Wilmington, MA) at 7 12 weeks of age were maintained in a pathogen-free environment, and all in vivo procedures were performed in strict accordance with the NIH Guide for the Care and Use of Laboratory Animals and approved by the Synta Pharmaceuticals Corp. Institutional Animal Care and Use Committee. NCI-HCC827, NCI-H1975, NCI-H1666, or NCI-H322 cells $\left(5 \times 10^{6}\right)$ were subcutaneously implanted into SCID mice. Mice bearing established tumors $\left(100-200 \mathrm{~mm}^{3}\right)$ were allocated into treatment groups of eight $(n=4$ for the NCI-H1666 experiment) exhibiting similar average tumor volumes and dosed either vehicle, ganetespib (i.v.) formulated in DRD (10 \% DMSO, $18 \%$ Cremophor RH 40, $3.6 \%$ dextrose), erlotinib (p.o.) formulated in $0.1 \%$ Tween in PBS, or afatinib (p.o.) formulated in DRD, using the schedules and regimens indicated. Tumor volumes $(V)$ were calculated by caliper measurements of the width $(W)$, length $(L)$, and thickness $(T)$ of each tumor using the formula: $V=0.5236$ (LWT). Tumor growth inhibition was determined from the change in average tumor volumes of each treated group relative to the vehicletreated, or itself in the case of tumor regression. Statistical significance was determined using two-way ANOVA followed by Bonferroni post tests. 


\section{Results}

Ganetespib potentiates the activity of TKIs and overcomes erlotinib resistance in NSCLC tumors driven by activating EGFR mutations

We and others have previously shown that ganetespib exhibits robust cytotoxic activity against NSCLC lines harboring a spectrum of activating EGFR mutations, and moreover, ganetespib retains potent activity against erlotinib-resistant NSCLC tumor phenotypes in vitro $[28,34]$. To extend these findings, here we sought to determine whether concurrent ganetespib exposure could potentiate EGFR TKI activity in vivo. Initially, SCID mice bearing NCI-HCC827 xenografts were dosed with ganetespib and erlotinib, either alone or in combination, on a weekly dosing schedule (Fig. 1a). NCIHCC 827 cells express a mutationally activated EGFR $^{\text {Del }}$ E746_A750 receptor and are sensitive to erlotinib treatment. As expected, weekly administration of erlotinib at $50 \mathrm{mg} / \mathrm{kg}$ was highly efficacious, resulting in $11 \%$ tumor regression. Although an equivalent dose of ganetespib only inhibited tumor growth by $67 \%$ (i.e., T/C value, $33 \%$ ), concurrent administration of ganetespib with erlotinib significantly improved the TKI antitumor response, inducing $67 \%$ tumor regression $(p=$ 0.0003 ). All treatments were well tolerated, with no significant loss of body weights observed over the 5 weeks of dosing (Fig. 1b).

This capacity to potentiate the activity of erlotinib therefore prompted an evaluation of combination therapy using the erlotinib-resistant, EGFR ${ }^{\text {L858R/T790M }}$ mutant-expressing NCI-H1975 NSCLC model. At the molecular level, singleagent ganetespib treatment resulted in a potent and dosedependent destabilization of EGFR ${ }^{\mathrm{L} 858 \mathrm{R} / \mathrm{T} 790 \mathrm{M}}$ and HER2 in NCI-H1975 cells (Fig. 2a). This was accompanied by

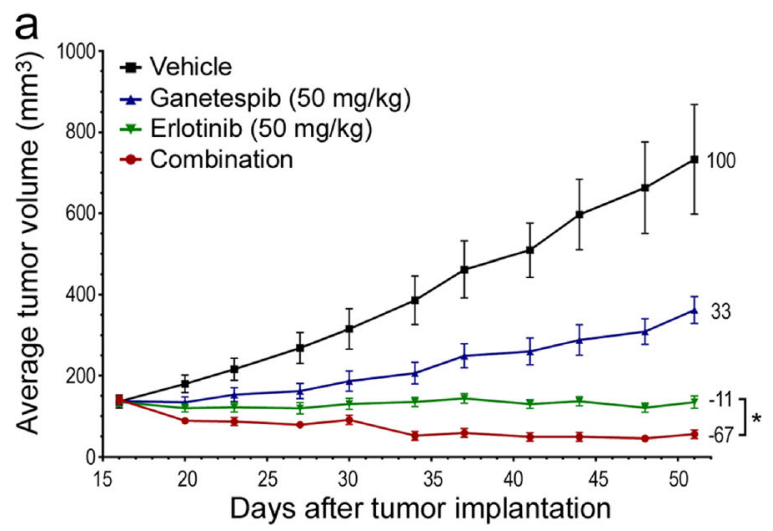

Fig. 1 Ganetespib confers superior antitumor efficacy in combination with erlotinib in NCI-HCC827 NSCLC xenografts. a Mice bearing NCIHCC 827 tumors ( $n=8$ mice/group) were i.v. administered ganetespib or p.o. dosed with erlotinib (each at $50 \mathrm{mg} / \mathrm{kg}$ ) on a weekly dosing regimen, either alone or in combination. Data are expressed as mean and SEM for each time point. Numerical T/C values are indicated to the right of each inactivation of downstream effector pathways including STAT signaling, the mitogen-activated protein kinase (MAPK) pathway, and AKT activity, as evidenced by loss of (phosphorylated) p-STAT3, p-ERK, and p-AKT protein expression, respectively. These effects were associated with a concomitant increase in BIM protein levels, a marker of apoptosis (Fig. 2a). In vivo, erlotinib dosed at its maximally tolerated dose (MTD) of $200 \mathrm{mg} / \mathrm{kg}$ had no antitumor activity, consistent with the resistant phenotype of these cells (Fig. 2b). Ganetespib (50 mg/kg) monotherapy was moderately efficacious, inhibiting tumor growth by $39 \%$ (T/C value, $61 \%$ ). However, when the two agents were combined, a significant improvement in efficacy was seen (T/C, $28 \% ; p<0.0001)$. This combinatorial benefit suggested that ganetespib cotreatment was sufficient to overcome erlotinib resistance in these tumors.

Next, we evaluated combinations of ganetespib with afatinib [35], which has reported antiproliferative activity in preclinical models expressing T790M [36] and was recently approved as a first-line oral treatment for NSCLC with EGFR exon 19 deletions or exon 21 (L858R) substitution mutations. In vitro, treatment of NCI-H1975 cells with $100 \mathrm{nM}$ ganetespib resulted in robust degradation of EGFR protein expression and concomitant reductions in p-EGFR activity, as well as loss of activated (phosphorylated) forms of MEK and ERK. Consistent with its TKI mode of action, afatinib reduced EGFR activity (as evidenced by reduced p-EGFR expression) without affecting total receptor levels (Fig. 2c). Combination treatment using a constant dose of ganetespib (100 nM) with increasing concentrations of afatinib indicated that ganetespib exposure could potentiate the activity of the TKI, sufficient to cause complete abrogation of EGFR activity and downstream effector signaling (Fig. 2c). Interestingly, combination treatment appeared to partially stabilize total EGFR protein levels

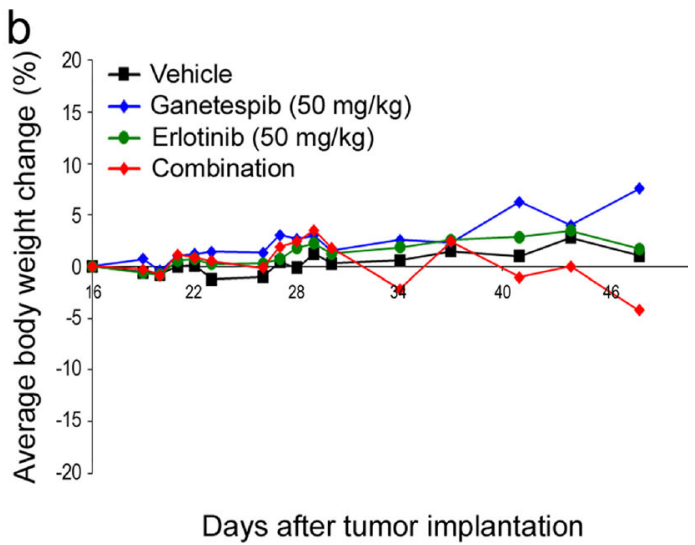

growth curve. Combinatorial ganetespib plus erlotinib therapy was significantly more efficacious than either agent alone (combination vs. erlotinib, $\left.{ }^{*} p=0.0003\right)$. b Body weights were measured five times per week for the first 2 weeks of dosing and twice per week thereafter. Mean values are plotted against vehicle controls 
a

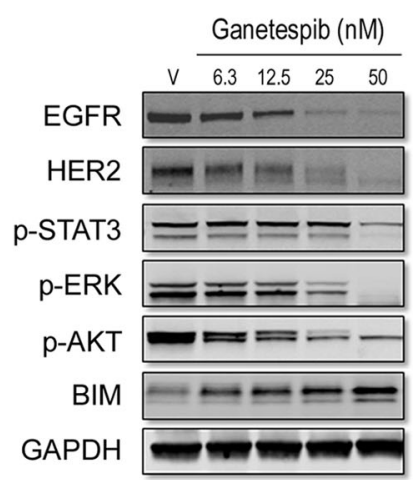

C

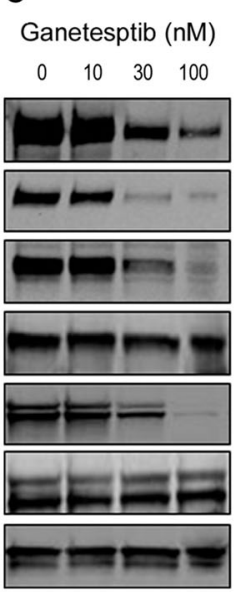

Ganetespib (100 nM) + Afatinib (nM)

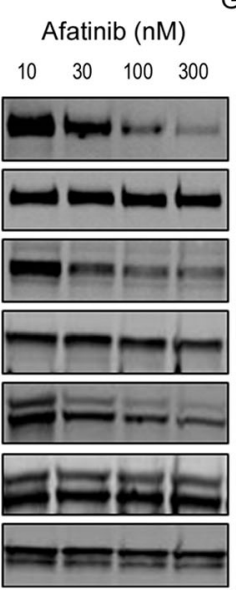

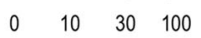

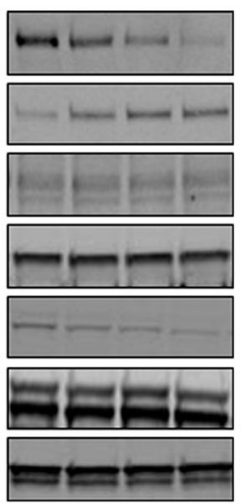

p-EGFR

EGFR

p-MEK

MEK

$p$-ERK

ERK

GAPDH
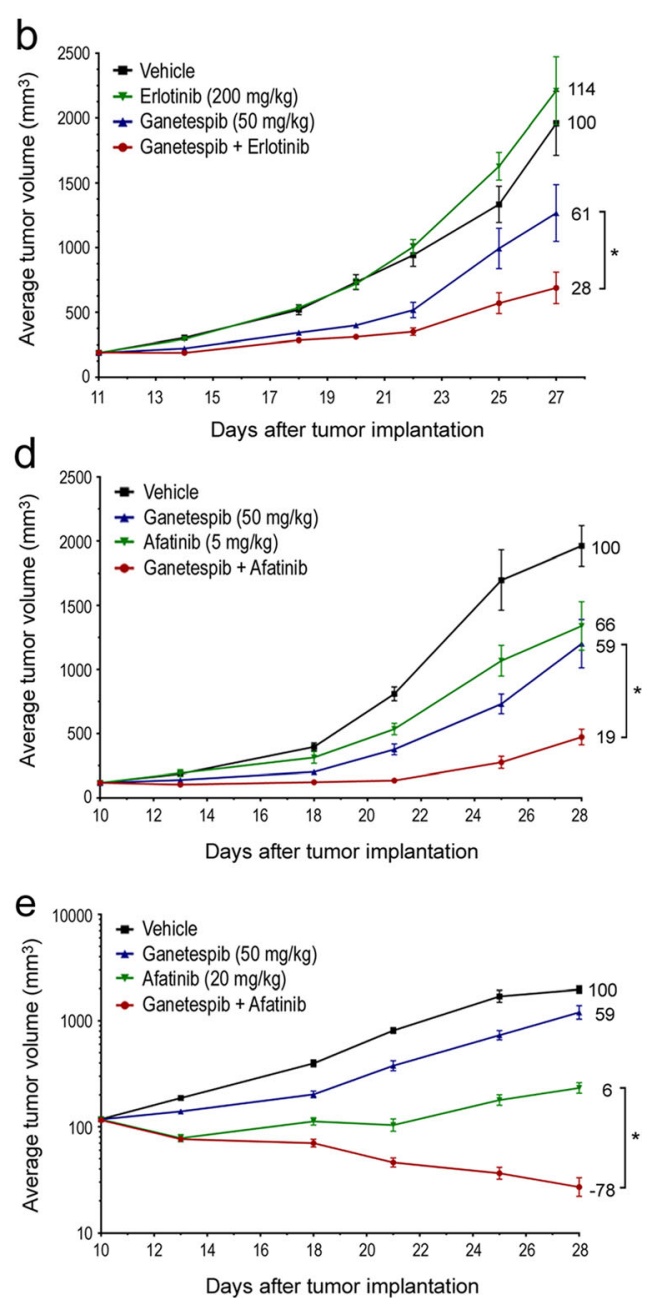

were incubated with the indicated concentrations of ganetespib or afatinib, both alone and in combination, for $24 \mathrm{~h}$. Cell lysates were analyzed by Western blotting. d Mice bearing NCI-H1975 tumors $(n=8$ mice/group) were i.v. dosed with ganetespib $(50 \mathrm{mg} / \mathrm{kg})$ once weekly and afatinib (5 mg/kg) administered p.o. five times/week, either alone or in combination. Combinatorial ganetespib plus afatinib therapy was significantly more efficacious than either agent alone $(* p<0.001)$. e Mice bearing NCI-H1975 tumors ( $n=8$ mice/group) were i.v. dosed with ganetespib $(50 \mathrm{mg} / \mathrm{kg})$ once weekly and afatinib $(20 \mathrm{mg} / \mathrm{kg})$ administered p.o. five times/week, either alone or in combination. The combination of ganetespib and erlotinib was significantly more efficacious than singleagent therapy, resulting in tumor regressions $\left({ }^{*} p<0.0001\right)$

(Fig. 2d). Moreover, ganetespib co-treatment significantly potentiated afatinib activity even at a more efficacious dose of the TKI (Fig. 2e). Afatinib dosed at $20 \mathrm{mg} / \mathrm{kg}$ resulted in effective stabilization of NCI-H1975 xenograft tumor growth $(\mathrm{T} / \mathrm{C}, 6 \%)$, with dual therapy causing $78 \%$ tumor regression $(p<0.0001)$. All treatments were well tolerated, with no significant toxicity or loss of body weights observed over the course of the dosing regimen (Supplementary Fig. S1). Taken together, these data indicated that ganetespib possesses potent sensitizing properties when combined with standard of care TKI drugs in EGFR mutant-driven NSCLC. 
Characterizing ganetespib activity in WT-EGFR NSCLC models

To investigate ganetespib activity within the context of the WT-EGFR phenotype, the cytotoxic activity of the compound was first evaluated using a panel of 31 WT-EGFR-expressing NSCLC lines, where it reduced cell viability with low nanomolar potency (Table 1). Our analysis included the NCI-H1838 cell line which exhibits genomic amplification of EGFR, resulting in overexpression of the wild-type receptor [37]. Ganetespib had a cytotoxicity $\mathrm{IC}_{50}$ value in these cells of $21 \mathrm{nM}$, virtually identical to the overall median value for all the lines examined (22 $\mathrm{nM})$. Expression changes in HSP90 client proteins and signaling pathways associated with NSCLC progression were then investigated using the ganetespib-sensitive, WT-EGFR cell line NCI-H1666. As expected, targeted degradation of both EGFR and HER2 receptor expression was induced in a dose-dependent manner, with maximal effects achieved at drug concentrations of $100 \mathrm{nM}$ or greater (Fig. 3a). Of note, NCI-H1666 cells harbor a mutant $\mathrm{BRAF}^{\mathrm{G} 466 \mathrm{~V}}$ kinase. Mutant BRAF proteins are particularly reliant on HSP90 activity for stability, and accordingly, destabilization of BRAF ${ }^{\mathrm{G} 466 \mathrm{~V}}$ protein levels also occurred following ganetespib treatment. However, in contrast
Fig. 3 Ganetespib inhibits oncogenic signaling and suppresses tumor growth in WTEGFR NSCLC models. a NCIH1666 cells were incubated with the indicated concentrations of ganetespib for $24 \mathrm{~h}$. Cell lysates were analyzed by Western blotting. b NCI-H1666 cells were treated with $100 \mathrm{nM}$ ganetespib for the indicated time periods. Cell lysates were analyzed by Western blotting. c Mice bearing NCI-H1666 tumors $(n=4$ mice/group) were i.v. administered ganetespib $(150 \mathrm{mg} / \mathrm{kg})$ on a weekly dosing regimen for 3 weeks. Data are expressed as mean and SEM for each time point. Numerical T/C values are indicated to the right of each growth curve
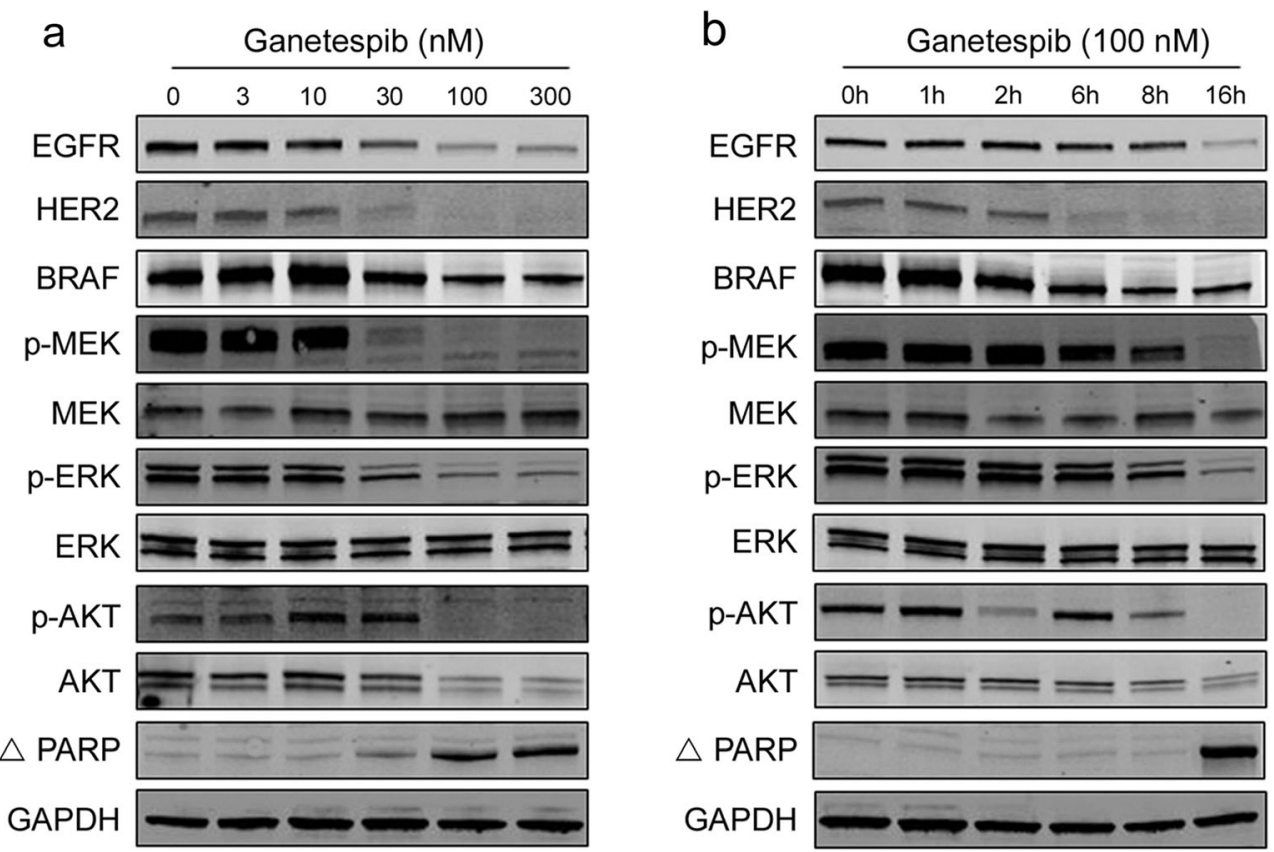

\section{C}

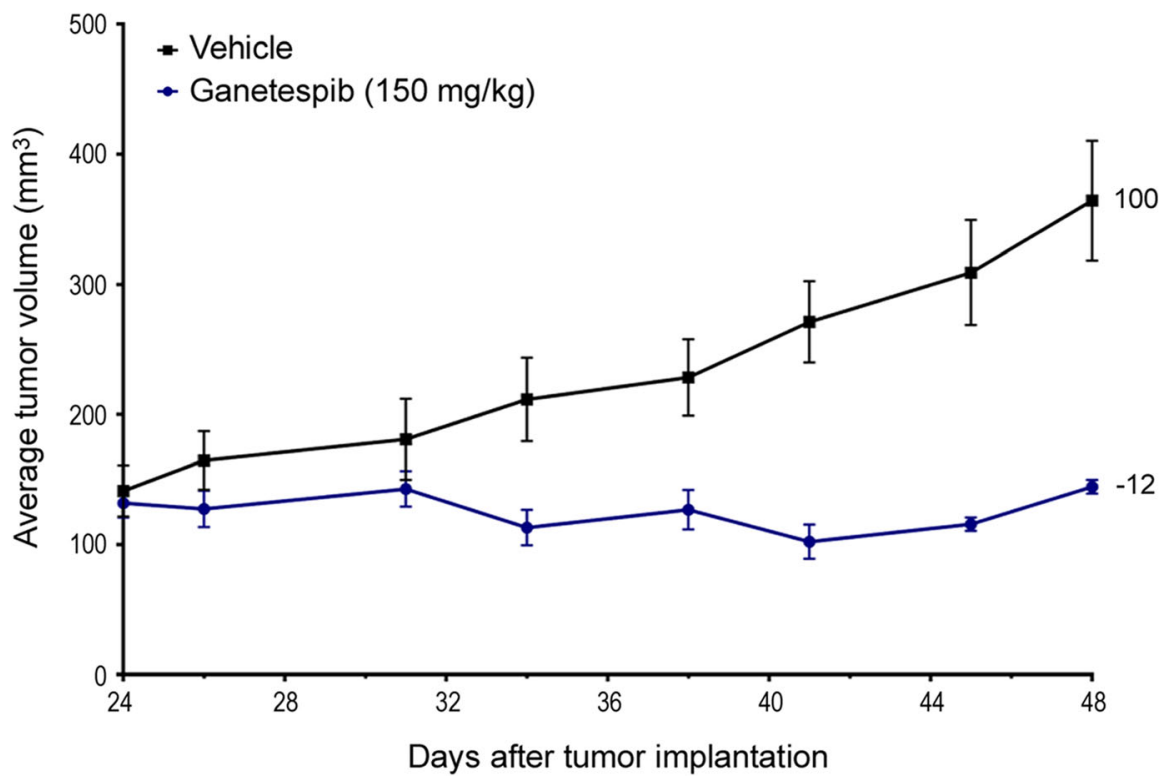


to most other BRAF mutations (e.g., $\mathrm{BRAF}^{\mathrm{V} 600 \mathrm{E}}$ ) which are activating and confer transforming capacity, $\mathrm{BRAF}^{\mathrm{G} 466 \mathrm{~V}}$ is kinase impaired [38] and thus not likely to be a primary driver of oncogenesis in this cell line. The coordinate impacts on these upstream signaling mediators arising from ganetespib treatment produced convergent effects on MAPK and survival pathway signaling (Fig. 3a). This was demonstrated by the complete loss of active (phosphorylated) MEK and AKT expression, as well as significantly reduced ERK activity, seen at comparable drug concentrations. These molecular changes correlated with a robust increase in cleaved poly(ADP-ribose) polymerase (PARP) expression, another marker of apoptosis (Fig. 3a).

When the kinetics of client pathway modulation in response to HSP90 inhibition were examined (Fig. 3b), it was found that 100-nM ganetespib treatment promoted the destabilization of WT-EGFR which was most evident at the 16-h time point. By comparison, a more rapid loss of HER2 protein expression was achieved, occurring by $6 \mathrm{~h}$. These data are in agreement with the differential sensitivity of the two receptors to pharmacological HSP90 blockade. Interestingly, we observed a transient rebound in p-AKT expression between 2 and $6 \mathrm{~h}$ following ganetespib exposure; however, this cellular response was effectively suppressed in the continued presence of the HSP90 inhibitor. Overall, the kinetics for maximal reductions in MEK, ERK, and AKT activities matched those observed for the elevation of cleaved PARP levels (Fig. 3b).

To determine whether these effects on viability and cell signaling translated to antitumor activity in vivo, we evaluated the single-agent efficacy of ganetespib treatment on the growth of NCI-H1666 xenografts. As shown in Fig. 3c, mice bearing NCI-H1666 tumors that were treated on a weekly dosing schedule of ganetespib at its MTD of $150 \mathrm{mg} / \mathrm{kg}$ [28]

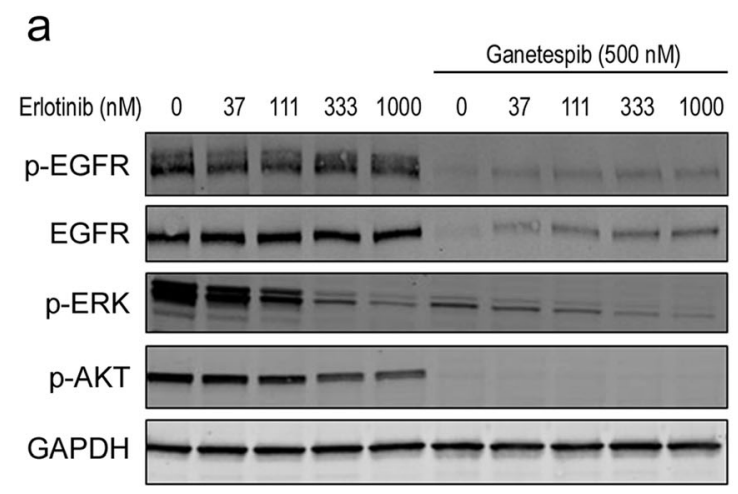

Fig. 4 Ganetespib potentiates the activity of erlotinib to induce tumor regression in WT-EGFR NSCLC. a NCI-H322 cells were incubated with the graded concentrations of erlotinib for $24 \mathrm{~h}$, with or without $500 \mathrm{nM}$ ganetespib. Cell lysates were analyzed by Western blotting. b Mice bearing NCI-H322 tumors ( $n=8$ mice/group) were i.v. dosed with ganetespib $(50 \mathrm{mg} / \mathrm{kg})$ once weekly and erlotinib $(25 \mathrm{mg} / \mathrm{kg})$ exhibited a significant decrease in tumor growth, resulting in $12 \%$ tumor regression. Thus, the potent and durable loss of client protein expression and perturbation of oncogenic signaling induced by ganetespib exposure effectively suppressed tumor growth in this WT-EGFR NSCLC model.

Combination ganetespib plus erlotinib treatment induces tumor regressions in WT-EGFR NSCLC

Combinatorial erlotinib plus ganetespib treatment was subsequently evaluated in an additional NSCLC model of WTEGFR background. To minimize any confounding oncogenic driver effects, the NCI-H322 cell line was chosen for these experiments as it is wild-type for EGFR, BRAF, as well as KRAS. Consistent with its mode of action, erlotinib alone had no effect on total EGFR protein levels but did reduce EGFR phosphorylative status in NCI-H322 cells (Fig. 4a). At the highest dose levels examined, a reduction in ERK activity (i.e., p-ERK expression) and modest impact on AKT signaling were observed. A high dose of ganetespib $(500 \mathrm{nM})$ was sufficient to effectively degrade EGFR protein levels (inducing a concomitant loss of p-EGFR activity), abrogate AKT signaling, and reduce, although not completely inhibit, p-ERK expression in NCI-H322 cells. In the presence of ganetespib, and similar to what was observed for the ganetespib/afatinib combination in Fig. 2c, concurrent treatment with increasing concentrations of erlotinib partially stabilized EGFR protein levels in a dose-dependent manner. Despite this, no reactivation of EGFR kinase activity, as evidenced by p-EGFR expression, occurred with concomitant ganetespib exposure. In addition, ERK signaling activity was further suppressed in an erlotinib-dependent manner in combination-treated NCI-H322 cells (Fig. 4a). Similar

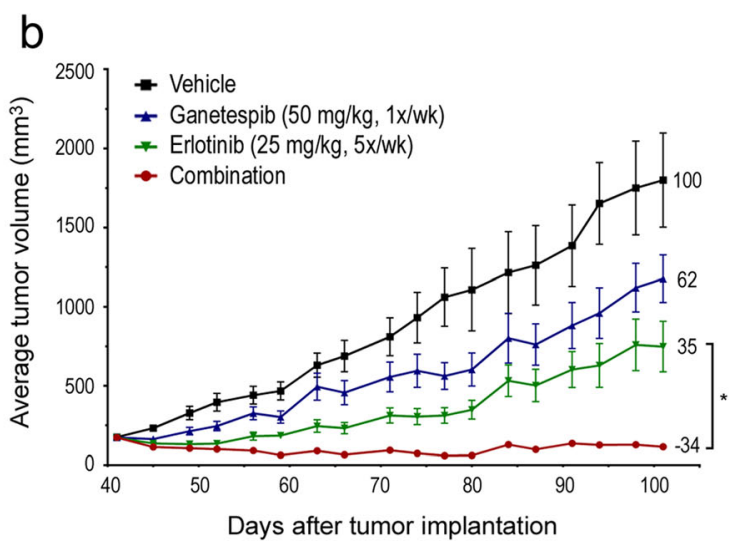

administered p.o. five times/week, either alone or in combination. Numerical $\mathrm{T} / \mathrm{C}$ values are indicated to the right of each growth curve, and the errors bars are the SEM. The combination of ganetespib and erlotinib was significantly more efficacious than either agent alone, resulting in tumor regressions (combination vs. erlotinib, ${ }^{*} p<0.0001$ ) 
molecular effects and pathway modulation were observed in another WT-EGFR cell line, NCI-H292 (Supplementary Fig. S2).

Finally, we performed an extended in vivo analysis of combination ganetespib plus erlotinib treatment using NCIH322 xenografts. Mice bearing NCI-H322 tumors were treated with a one third MTD $(50 \mathrm{mg} / \mathrm{kg})$ weekly dose of ganetespib or erlotinib $(25 \mathrm{mg} / \mathrm{kg})$ five times/week, either alone or in combination, over a 2-month treatment period (Fig. 4b). Ganetespib monotherapy resulted in $38 \%$ tumor growth inhibition, while erlotinib suppressed tumor growth by $65 \%$. When administered concurrently, a significant improvement in antitumor efficacy was achieved, resulting in $34 \%$ tumor regression. These data clearly demonstrate that the therapeutic sensitizing properties of ganetespib for direct EGFR kinase inhibition were conserved within the WTEGFR NSCLC setting.

\section{Discussion}

EGFR is a validated therapeutic target in NSCLC, with three small molecule TKIs (erlotinib, gefitinib, and afatinib) currently approved for the treatment of advanced disease [22]. All of these agents show preferential clinical efficacy in NSCLC patients whose tumors harbor activating EGFR mutations. Mutant EGFR oncoproteins are particularly reliant on the chaperone activity of HSP90 for their conformational stability and function [39, 40]. Thus, pharmacological blockade of HSP90, leading to the degradation and loss of mutant EGFR protein, is considered a rational and alternative strategy to TKI inhibition for treating tumors driven by such genetic modifications. Moreover, it has recently been demonstrated that the mature, wild-type receptor is also a bona fide HSP90 client in cancers that overexpress WT-EGFR [41], suggesting potential utility for targeted HSP90 inhibitors beyond the mutant receptor phenotype. Despite this, the clinical experience with ganetespib (and other selective HSP90 inhibitors) has revealed only modest single-agent activity in molecularly defined subsets of both wild-type and mutant EGFR NSCLC patients [32, 42]. It has been proposed that the full therapeutic benefit of small molecule inhibitors of HSP90 is likely to be realized when they are used as part of combinatorial approaches, alongside standard-of-care or other molecularly targeted agents [20]. In this regard, ganetespib has shown potent chemosensitizing activity when administered in conjunction with taxanes in NSCLC, in both the preclinical and clinical settings [30,33]. These considerations thus prompted the comprehensive evaluation of combining the modalities of HSP90 blockade with selective EGFR tyrosine kinase inhibition presented here.
Initially, we performed analyses using xenograft tumors derived from the mutant $\mathrm{EGFR}^{\mathrm{Del}}{ }^{\mathrm{E} 746}{ }^{\mathrm{A}}{ }^{750}$-driven NCIHCC 827 NSCLC cell line, wherein the presence of this particular mutation confers sensitivity to erlotinib exposure. Indeed, erlotinib treatment was highly efficacious in vivo, resulting in measurable tumor regressions and disease stabilization. Since mutated EGFR is an established HSP90 client and NCI-HCC827 cells are sensitive to ganetespib exposure [28], we selected a low (one third MTD) dose of ganetespib for these experiments in order to readily permit evaluation of potential combinatorial improvements in efficacy between the two drugs. As a single agent, ganetespib displayed only modest antitumor activity at this dose level. However, when combined with erlotinib, ganetespib greatly improved the therapeutic response, significantly enhancing the overall degree of regression in this model. The molecular basis underlying the superior efficacy remains to be fully elucidated. However, it is reasonable to suggest that coordinate impacts on both the signaling activity and functional stability of EGFR ${ }^{\text {Del E746_A750 (afforded }}$ by selective kinase and HSP90 inhibition, respectively) promoted a more robust and durable loss of EGFR oncogenic driver activity that resulted in greater tumor shrinkage. Further, the pleiotropic effects of HSP90 inhibition would also be expected to impact convergent and/or parallel signaling pathways to EGFR; this broader spectrum of biologic activity might also contribute to the improved therapeutic indices.

While initially effective in appropriately selected NSCLC populations, durable responses to erlotinib are rare, and most patients invariably progress to drug-resistant disease, typically within around 12 months [43]. The predominant mechanism of acquired resistance, accounting for approximately half of all cases, is the acquisition of a secondary mutation (T790M) at the gatekeeper residue of EGFR [43]. Indeed, the higher prevalence of this mutation in patients previously treated with an EGFR TKI indicates that TKI therapy itself provides a strong selection pressure for this molecular alteration and consequent development of resistance [44]. We have previously reported that erlotinib-resistant, EGFR ${ }^{\mathrm{L} 858 \mathrm{R} / \mathrm{T} 790 \mathrm{M}}$ mutant-expressing NCI-H1975 cells display sensitivity to ganetespib [28] and, as we show here, this results from potent destabilization and loss of EGFR ${ }^{\mathrm{L} 858 \mathrm{R} / \mathrm{T} 790 \mathrm{M}}$ receptor expression, with concomitant effects on downstream effector pathways including STAT, ERK, and AKT signaling. This is consistent with the premise that tumors that have become erlotinib-resistant via secondary T790M mutation are still dependent on aberrant EGFR activity for their continued growth and survival. As expected, NCI-H1975 xenograft tumors were insensitive to erlotinib treatment, and a one third MTD dose of ganetespib resulted in a modest degree of tumor growth inhibition when administered as monotherapy. However, concurrent treatment with both drugs resulted in a significantly improved antitumor response, indicating that dual ganetespib+erlotinib therapy may provide a means to 
overcome erlotinib resistance in this clinically relevant NSCLC model. This result is in agreement with a preclinical study showing similar combinatorial benefit when erlotinib was combined with an investigational HSP90 inhibitor compound CH5164840 [45]. Encouraging signs of clinical activity with the HSP90 inhibitor AUY922, dosed in combination with erlotinib, in patients with acquired resistance to EGFR inhibitors was recently reported from a phase II study (NCT01259089) [46]. Five of 22 patients demonstrated a partial response; three of which had the T790M mutation. Together, the data suggest that HSP90 inhibition alongside EGFR kinase blockade may represent an effective drug combination for overcoming TKI resistance in EGFR-mutant NSCLC.

One approach to counteract T790M-mediated resistance mechanisms has been the development of irreversible TKIs, such as the dual EGFR/HER inhibitor afatinib. Unlike erlotinib and gefitinib, which are reversible ATP-competitive agents, afatinib covalently binds to EGFR to irreversibly block receptor tyrosine kinase activity [35]. Accordingly, afatinib has shown robust activity in preclinical models against tumor lines harboring the T790M mutation, including NCI-H1975 [36]. Interestingly, our study also uncovered a significant enhancement of afatinib antitumor efficacy in response to ganetespib co-treatment in these xenograft tumors. At suboptimal dose levels that each induced a similar degree of tumor growth inhibition as single agents, combination treatment of ganetespib with afatinib resulted in a significant enhancement of tumor suppression. Remarkably, even at a more efficacious afatinib dose that effectively inhibited tumor growth, the addition of ganetespib to the regimen promoted near-complete tumor regressions. While the precise mechanism(s) by which ganetespib potentiates the activity of EFGR TKIs of both the reversible and irreversible classes is currently under investigation, further evaluation of this combinatorial treatment strategy is warranted. In this regard, an early-stage clinical trial combining ganetespib with afatinib in NSCLC patients with EGFR-activating mutations and acquired erlotinib resistance is planned.

A significant finding of the present study was that the improved therapeutic efficacy afforded by combination ganetespib plus erlotinib treatment was also observed within the WT-EGFR NSCLC setting. These data also provided some insights to the molecular mechanisms underlying the interaction between kinase and HSP90 inhibition. While erlotinib treatment resulted in a degree of receptor stabilization, no reactivation of EGFR kinase activity was observed in the presence of ganetespib. In addition, ERK signaling appeared to represent a point of convergence between the two agents, given that ERK activity was completely suppressed in cells following dual drug treatment. In contrast to its established role as first-line therapy for NSCLC patients with EGFRmutated tumors, the use of erlotinib as a second- or third- line salvage treatment for WT-EGFR patients that have progressed on prior chemotherapy remains contentious [18]. For example, the recent TAILOR phase III trial comparing erlotinib with docetaxel in advanced NSCLC patients with WT-EGFR tumors for whom first-line platinum therapy had failed demonstrated a clear superiority for the standard chemotherapy arm [47]. However, the initial clinical evaluation of erlotinib was performed in unselected NSCLC populations, and retrospective analyses of those randomized trials has revealed that, while overall response rates were higher in patients with EGFR mutations, survival benefit from singleagent erlotinib treatment was maintained in a proportion of the wild-type population $[15,48]$. The underlying molecular basis for the modest, yet clinically meaningful, improvements in survival outcomes for WT-EGFR individuals is still unknown, and biomarkers predicting for TKI response in WT-EGFR patients are lacking [17]. Moreover, there is considerable interest in identifying strategies to both exploit and improve the efficacy of selective TKIs such as erlotinib in wild-type disease. To date, efforts to combine EGFR TKIs with standard chemotherapies have not been associated with survival benefits in clinical trials [5]. The data presented here suggest that the multimodal effects afforded by HSP90 inhibition may represent a novel approach for optimizing response to EGFR TKIs for the large majority of NSCLC cases and thus warrant further clinical investigation.

Overall, the capacity of ganetespib to potentiate the in vivo activity of selective EGFR TKIs (including erlotinib and afatinib) provides a compelling rationale for combining these agents as part of novel treatment strategies for NSCLC. Moreover, the therapeutic benefit conferred by dual HSP90/EGFRTKI blockade was conserved within both the mutant and WTEGFR disease settings - thus providing new opportunities to target EGFR and overcome mechanisms of resistance across a spectrum of NSCLC patients.

Acknowledgments The authors would like to thank Kevin Foley for his contribution to the experimental design during the genesis of these studies.

Conflict of interest Dr. Smith has nothing to disclose. Dr. Acquaviva has nothing to disclose. Dr. Sequeira has nothing to disclose. Dr. Jimenez has nothing to disclose. Dr. Zhang has nothing to disclose. Dr. Sang has nothing to disclose. Dr. Bates has nothing to disclose. Dr. Proia has nothing to disclose.

Ethical standards The manuscript does not contain clinical studies or patient data.

Open AccessThis article is distributed under the terms of the Creative Commons Attribution Noncommercial License which permits any noncommercial use, distribution, and reproduction in any medium, provided the original author(s) and the source are credited. 


\section{References}

1. Jemal A, Center MM, DeSantis C, Ward EM (2010) Global patterns of cancer incidence and mortality rates and trends. Cancer Epidemiol Biomarkers Prev 19:1893-1907

2. Siegel R, Naishadham D, Jemal A (2013) Cancer statistics, 2013. CA Cancer J Clin 63:11-30

3. Ramalingam S, Belani C (2008) Systemic chemotherapy for advanced non-small cell lung cancer: recent advances and future directions. Oncologist 13(Suppl 1):5-13

4. Grossi F, Kubota K, Cappuzzo F, de Marinis F, Gridelli C et al (2010) Future scenarios for the treatment of advanced non-small cell lung cancer: focus on taxane-containing regimens. Oncologist 15:11021112

5. Ramalingam SS, Owonikoko TK, Khuri FR (2011) Lung cancer: new biological insights and recent therapeutic advances. CA Cancer J Clin 61:91-112

6. Gaughan EM, Costa DB (2011) Genotype-driven therapies for nonsmall cell lung cancer: focus on EGFR, KRAS and ALK gene abnormalities. Ther Adv Med Oncol 3:113-125

7. Herbst RS, Lynch TJ, Sandler AB (2009) Beyond doublet chemotherapy for advanced non-small-cell lung cancer: combination of targeted agents with first-line chemotherapy. Clin Lung Cancer 10: 20-27

8. Stella GM, Luisetti M, Inghilleri S, Cemmi F, Scabini R et al (2012) Targeting EGFR in non-small-cell lung cancer: lessons, experiences, strategies. Respir Med 106:173-183

9. Scagliotti GV, Selvaggi G, Novello S, Hirsch FR (2004) Clin Cancer Res $10: 4227 \mathrm{~s}-4232 \mathrm{~s}$

10. Ono M, Kuwano M (2006) Molecular mechanisms of epidermal growth factor receptor (EGFR) activation and response to gefitinib and other EGFR-targeting drugs. Clin Cancer Res 12:72427251

11. Sharma SV, Bell DW, Settleman J, Haber DA (2007) Epidermal growth factor receptor mutations in lung cancer. Nat Rev Cancer 7 : 169-181

12. Kobayashi S, Boggon TJ, Dayaram T, Janne PA, Kocher O et al (2005) EGFR mutation and resistance of non-small-cell lung cancer to gefitinib. N Engl J Med 352:786-792

13. Pao W, Miller VA, Politi KA, Riely GJ, Somwar R et al (2005) Acquired resistance of lung adenocarcinomas to gefitinib or erlotinib is associated with a second mutation in the EGFR kinase domain. PLoS Med 2:e73

14. Carrera S, Buque A, Azkona E, Aresti U, Calvo B et al (2014) Epidermal growth factor receptor tyrosine-kinase inhibitor treatment resistance in non-small cell lung cancer: biological basis and therapeutic strategies. Clin Transl Oncol 16:339-350

15. Jazieh AR, Al Sudairy R, Abu-Shraie N, Al Suwairi W, Ferwana M et al (2013) Erlotinib in wild type epidermal growth factor receptor non-small cell lung cancer: a systematic review. Ann Thorac Med 8: 204-208

16. Gazdar AF (2009) Activating and resistance mutations of EGFR in non-small-cell lung cancer: role in clinical response to EGFR tyrosine kinase inhibitors. Oncogene 28(Suppl 1):S24-31

17. Laurie SA, Goss GD (2013) Role of epidermal growth factor receptor inhibitors in epidermal growth factor receptor wild-type non-smallcell lung cancer. J Clin Oncol 31:1061-1069

18. Gelsomino F, Agustoni F, Niger M, Valota M, Haspinger ER (2013) Epidermal growth factor receptor tyrosine kinase inhibitor treatment in patients with EGFR wild-type non-small-cell lung cancer: the never-ending story. J Clin Oncol 31:3291-3293

19. Jassem J, Dziadziuszko R (2013) EGFR inhibitors for wild-type EGFR NSCLC: to use or not to use? Lancet Oncol 14:916-917

20. Neckers L, Workman P (2012) Hsp90 molecular chaperone inhibitors: are we there yet? Clin Cancer Res 18:64-76
21. Taipale M, Jarosz DF, Lindquist S (2010) HSP90 at the hub of protein homeostasis: emerging mechanistic insights. Nat Rev Mol Cell Biol 11:515-528

22. Reungwetwattana T, Dy GK (2013) Targeted therapies in development for non-small cell lung cancer. J Carcinog 12:22

23. Whitesell L, Lindquist SL (2005) HSP90 and the chaperoning of cancer. Nat Rev Cancer 5:761-772

24. Trepel J, Mollapour M, Giaccone G, Neckers L (2010) Targeting the dynamic HSP90 complex in cancer. Nat Rev Cancer 10:537-549

25. Xu W, Neckers L (2007) Targeting the molecular chaperone heat shock protein 90 provides a multifaceted effect on diverse cell signaling pathways of cancer cells. Clin Cancer Res 13:1625-1629

26. Banerji U (2009) Heat shock protein 90 as a drug target: some like it hot. Clin Cancer Res 15:9-14

27. Neckers L (2007) Heat shock protein 90: the cancer chaperone. J Biosci 32:517-530

28. Ying W, Du Z, Sun L, Foley KP, Proia DA et al (2012) Ganetespib, a unique triazolone-containing Hsp90 inhibitor, exhibits potent antitumor activity and a superior safety profile for cancer therapy. Mol Cancer Ther 11:475-484

29. Proia DA, Bates RC (2014) Ganetespib and HSP90: translating preclinical hypotheses into clinical promise. Cancer Res 74:1294 1300

30. Proia DA, Sang J, He S, Smith DL, Sequeira M et al (2012) Synergistic activity of the Hsp90 inhibitor ganetespib with taxanes in non-small cell lung cancer models. Investig New Drug 30:22012209

31. Acquaviva J, Smith DL, Sang J, Friedland JC, He S et al (2012) Targeting KRAS-mutant non-small cell lung cancer with the Hsp90 inhibitor ganetespib. Mol Cancer Ther 11:2633-2643

32. Socinski MA, Goldman J, El-Hariry I, Koczywas M, Vukovic V et al (2013) A multicenter phase II study of ganetespib monotherapy in patients with genotypically defined advanced non-small cell lung cancer. Clin Cancer Res 19:3068-3077

33. Ramalingam S, Goss GD, Andric ZG, Bondarenko I, Zaric B, et al (2013) A randomized study of ganetespib, a heat shock protein 90 inhibitor, in combination with docetaxel versus docetaxel alone for second-line therapy of lung adenocarcinoma (GALAXY-1). J Clin Oncol 31:abstr CRA8007

34. Shimamura T, Perera SA, Foley KP, Sang J, Rodig SJ et al (2012) Ganetespib (STA-9090), a nongeldanamycin HSP90 inhibitor, has potent antitumor activity in in vitro and in vivo models of non-small cell lung cancer. Clin Cancer Res 18:4973-4985

35. Solca F, Dahl G, Zoephel A, Bader G, Sanderson M et al (2012) Target binding properties and cellular activity of afatinib (BIBW 2992), an irreversible ErbB family blocker. J Pharmacol Exp Ther $343: 342-350$

36. Li D, Ambrogio L, Shimamura T, Kubo S, Takahashi M et al (2008) BIBW2992, an irreversible EGFR/HER2 inhibitor highly effective in preclinical lung cancer models. Oncogene 27:4702-4711

37. Yauch RL, Januario T, Eberhard DA, Cavet G, Zhu W et al (2005) Epithelial versus mesenchymal phenotype determines in vitro sensitivity and predicts clinical activity of erlotinib in lung cancer patients. Clin Cancer Res 11:8686-8698

38. Sen B, Peng S, Tang X, Erickson HS, Galindo H et al (2012) Kinaseimpaired BRAF mutations in lung cancer confer sensitivity to dasatinib. Sci Transl Med 4:136ra170

39. Shimamura T, Lowell AM, Engelman JA, Shapiro GI (2005) Epidermal growth factor receptors harboring kinase domain mutations associate with the heat shock protein 90 chaperone and are destabilized following exposure to geldanamycins. Cancer Res 65: 6401-6408

40. Shimamura T, Li D, Ji H, Haringsma HJ, Liniker E et al (2008) Hsp90 inhibition suppresses mutant EGFR-T790M signaling and overcomes kinase inhibitor resistance. Cancer Res $68: 5827-5838$ 
41. Ahsan A, Ramanand SG, Whitehead C, Hiniker SM, Rehemtulla A et al (2012) Wild-type EGFR is stabilized by direct interaction with HSP90 in cancer cells and tumors. Neoplasia 14:670-677

42. Sequist LV, Gettinger S, Senzer NN, Martins RG, Janne PA et al (2010) Activity of IPI-504, a novel heat-shock protein 90 inhibitor, in patients with molecularly defined non-small-cell lung cancer. J Clin Oncol 28:4953-4960

43. Sequist LV, Waltman BA, Dias-Santagata D, Digumarthy S, Turke AB et al (2011) Genotypic and histological evolution of lung cancers acquiring resistance to EGFR inhibitors. Sci Transl Med 3:75ra26

44. Su KY, Chen HY, Li KC, Kuo ML, Yang JC et al (2012) Pretreatment epidermal growth factor receptor (EGFR) T790M mutation predicts shorter EGFR tyrosine kinase inhibitor response duration in patients with non-small-cell lung cancer. J Clin Oncol 30:433-440
45. Ono N, Yamazaki T, Tsukaguchi T, Fujii T, Sakata K et al (2013) Enhanced antitumor activity of erlotinib in combination with the Hsp90 inhibitor CH5164840 against non-small-cell lung cancer. Cancer Sci 104:1346-1352

46. Johnson ML, Hart EM, Rademaker A, Weitner BB, Urman A, et al (2013) A phase II study of HSP90 inhibitor AUY922 and erlotinib (E) for patients (pts) with EGFR-mutant lung cancer and acquired resistance (AR) to EGFR tyrosine kinase inhibitors (EGFR TKIs). J Clin Oncol 31:abstr 8036

47. Garassino MC, Martelli O, Broggini M, Farina G, Veronese S et al (2013) Erlotinib versus docetaxel as second-line treatment of patients with advanced non-small-cell lung cancer and wild-type EGFR tumours (TAILOR): a randomised controlled trial. Lancet Oncol 14: 981-988

48. Piperdi B, Perez-Soler R (2012) Role of erlotinib in the treatment of non-small cell lung cancer: clinical outcomes in wild-type epidermal growth factor receptor patients. Drugs 72(Suppl 1):11-19 